\title{
Evolutionary consequences of a large duplication event in Trypanosoma brucei: Chromosomes 4 and 8 are partial duplicons Andrew P Jackson
}

Address: Wellcome Trust Sanger Institute, Wellcome Trust Genome Campus, Hinxton, Cambridgeshire. CB10 1SA. UK

Email: Andrew P Jackson - aj4@sanger.ac.uk

Published: 23 November 2007

BMC Genomics 2007, 8:432 doi:10.1/86/|47|-2/64-8-432
Received: 22 August 2007

Accepted: 23 November 2007

This article is available from: http://www.biomedcentral.com//47I-2/64/8/432

(c) 2007 Jackson; licensee BioMed Central Ltd.

This is an Open Access article distributed under the terms of the Creative Commons Attribution License (http://creativecommons.org/licenses/by/2.0), which permits unrestricted use, distribution, and reproduction in any medium, provided the original work is properly cited.

\begin{abstract}
Background: Gene order along the genome sequence of the human parasite Trypanosoma brucei provides evidence for a $0.5 \mathrm{Mb}$ duplication, comprising the 3' regions of chromosomes 4 and 8 . Here, the principal aim was to examine the contribution made by this duplication event to the $T$. brucei genome sequence, emphasising the consequences for gene content and the evolutionary change subsequently experienced by paralogous gene copies. The duplicated region may be browsed online at http://www.genedb.org/genedb/tryp/48dup image.jsp

Results: Comparisons of trypanosomatid genomes demonstrated widespread gene loss from each duplicon, but also showed that $47 \%$ of duplicated genes were retained on both chromosomes as paralogous loci. Secreted and surface-expressed genes were over-represented among retained paralogs, reflecting a bias towards important factors at the host-parasite interface, and consistent with a dosage-balance hypothesis. Genetic divergence in both coding and regulatory regions of retained paralogs was bimodal, with a deficit in moderately divergent paralogs; in particular, noncoding sequences were either conserved or entirely remodelled. The conserved paralogs included examples of remarkable sequence conservation, but also considerable divergence of both coding and regulatory regions. Sequence divergence typically displayed strong negative selection; but several features, such as asymmetric evolutionary rates, positively-selected codons and other nonneutral substitutions, suggested that divergence of some paralogs was driven by functional change. The absence of orthologs to retained paralogs in $T$. congolense indicated that the duplication event was specific to $T$. brucei.

Conclusion: The duplication of this chromosomal region doubled the dosage of many genes. Rather than creating 'more of the same', these results show that paralogs were structurally modified according to various evolutionary trajectories. The retention of paralogs, and subsequent elaboration of both their primary structures and regulatory regions, strongly suggests that this duplication was a seminal development, stimulating functional innovation and fundamentally altering the genetic repertoire of $T$. brucei relative to other trypanosomatids.
\end{abstract}

\section{Background}

The African trypanosome, Trypanosoma brucei, causes sleeping sickness and substantial human morbidity across Africa. The recently completed genome sequences for $T$. bruce i and two related protistan parasites, T. cruzi and Leishmania major [1-3], have provided a basis for understanding the biological and pathological differences among the Trypanosomatidae infecting humans. All 
trypanosomatid genomes share broad conservation of synteny, polycistronic transcription and a general absence of cis-introns (but isolated instances have been identified [2]). However, the number and size of chromosomes is known to vary between and within species, due to variation in repetitive, telomeric regions $[4,5]$ and the infrequent, irregular genetic exchange between strains [6-8]. The T. brucei haplotype includes 11 megabase-sized chromosomes, as well as numerous mini-chromosomes [9]; other species have many more indicating that T. brucei has experienced a sequence of chromosomal fusions [10]. This study shows that the T. brucei genome has acquired a previously unreported duplication affecting chromosomes 4 and 8, which does not reflect temporary karyotypic fluctuation. The report begins by documenting this partial chromosomal duplication to identify paralogous gene copies, and then a quantitative analysis of paralogous sequences examines the potential for evolutionary innovation and the importance of the duplication for the genomic repertoire in T. brucei.

The architecture of genome sequences has shown that duplication is a frequent and important process in genome evolution $[11,12]$. It occurs on every scale within the genome: mistakes during DNA synthesis cause tandem duplication of individual genes, and segmental duplication often results from the transposition of mobile elements; for instance, the transposition of an Alu element caused the duplication of the human BRCA1 region [13]. Duplication of whole chromosomes, or chromosomesized blocks, can result from mistakes during cell division, (i.e., non-disjunction). This can also occur after whole genome duplication (WGD) when a polyploid genome decays through selective gene loss. The relative importance of these processes seems to vary by gene function and taxon; WGD has been widely reported, most notably among plants [14-17] and yeasts [18-21], and may have been responsible for major evolutionary transitions in chordates [22-24]. Certainly, the prevalence and importance of duplication in genomic evolution has only recently become clear [11] and is among the major insights delivered by whole genome sequencing. These observations have helped to revive the argument of Ohno [25], eclipsed in the pre-genomic era by the focus on sequence evolution, that gene duplication is the principal source of evolutionary novelty [reviewed in [12]], faster and more consequential than nucleotide substitution.

The fate of gene duplicates seems to be multifarious and subject to various factors. Since duplications of any kind disrupt systems at, or near, optimality, one should assume that most duplications are selected against. Indeed, most loci created after large duplication events are subsequently deleted [12,18], resulting in 'diploidisation' in Arabidopsis thaliana for example $[22,26]$. However, gene loss is nei- ther complete nor random, and may show similar trends across taxa [27]; in teleost fishes, genes associated with signalling and gene regulation were enriched following gene loss [28], transcription factors were over-represented in rice [29], while $A$. thaliana preferentially retained signal transduction loci [26]. Loci with a high level of proteomic connectivity were also selectively retained in $A$. thaliana following WGD [26]. Ohno's original model [25] intuitively suggested that duplication facilitated novel functions (neofunctionalisation) through the relaxation of purifying selection due to redundancy after duplication. The importance of rapid, structural evolution to functional innovation has been inferred from the widespread asymmetry of evolutionary change among paralogous genes and regions [19,26,30-32], the acceleration of evolutionary rate among paralogs $[33,34]$ and positive selection of duplicated genes $[35,36]$.

In contrast to neofunctionalisation, functional change might result from a segregation of the original gene function between duplicates (subfunctionalisation), due to degenerate mutations in each, and producing copies with distinct specificities [37-40]. The duplication-degeneration-complementation model [38] refined this concept, stating that complementary mutations in regulatory regions were responsible for partitioning functionality. Many examples of duplicates performing generic functions, but with specific spatial or temporal expression profiles, are known; $m y b$-homologs in maize (Zea mays) are tandem duplicates and are expressed in distinct flower tissues due to divergence in their 3' regulatory regions [41]. In pufferfish (Takifugu rubripes), two copies of a Hox gene (Hoxa2) formed after WGD are expressed in distinct regions of the hindbrain, whereas their common ortholog in tetrapods is expressed throughout; tissue specificity evolved through changes in cis regulatory modules [42]. Along with loss of function (pseudogenisation, or nonfunctionalisation), a gallery of potential fates has been formulated. What is clear is that sensible changes in structure are correlated with changes in function; these may affect coding sequences or regulatory modules, and the precise outcome of duplication likely reflects both selective pressures (adaptation) and historical constraints, that is, the function, indispensability and connectivity of the original gene [43]. Duplicates may evolve through structural divergence or rapid changes in expression profile, but duplication always creates evolutionary opportunities, some of which may lead to novelty.

This study provides the first account of a large duplication event in T. brucei, which, if its consequences are similar to those in other organisms, may provide, or have provided, the raw material for evolutionary innovation and the expansion of gene families. Previously, studies of tandem gene arrays had documented gene duplication in trypano- 
somatids, for example, the phosphoglycerate kinase gene array [44] and hexose transporters [45], where gene duplication combined with gene conversion to create novel sequence types; this phenomenon is now known to be widespread [46]. Elaboration of important trypanosomatid gene families, such as amastin surface antigens in Leishmania spp. [47] and VSGs in T. brucei [48] are also the consequences of gene duplication. However, the impact of this chromosomal duplication has been much greater and, by examining the evolutionary changes that have subsequently affected each duplicon, this study sought to establish its contribution to the T. brucei genome. There were four specific aims: i) to document gene losses and gains since the duplication event; ii) to describe patterns of both coding and non-coding sequence divergence between paralogous gene pairs ; iii) to assess evidence for disparity in evolutionary rate during divergence using relative rates tests; iv) to assess the role of non-neutral substitutions in deriving new functions.

\section{Results \\ (a) Partial chromosomal duplication: gene content and order}

Comparison of gene order along Chromosomes 4 and 8 of $T$. brucei with homologous regions in T. cruzi and $L$. major demonstrated widespread colinearity within and between species. Figure 1 shows tBLASTx analyses between Chromosomes 4 and 8 and Chromosome 31 in L. major, visualised by the Artemis Comparison Tool (ACT). The blast hits between chromosomes 4 and 8 are given in greater detail in Additional data file 1. On Chromosome 8 (0.98-1.47 Mb), the duplicon begins with a ser/thr-protein kinase NrkAgene (1: Tb927.8.6930; paralogous gene pairs are numbered 1 to 74 and referred to by their GeneDB identifier tags), which is preceded upstream by a strand-switch region, and ends at the chromosomal terminus with a receptor-type adenylate cyclase (74: Tb927.8.8360). On Chromosome 4 (2-2.48 Mb), a para$\log$ of the ser/thr-protein kinase NrkAgene is found near the chromosomal terminus (1: Tb927.4.5390) and is followed downstream by a strand-switch region and several genes typical of T. brucei sub-telomeric regions. Synteny with Chromosome 8 is conserved upstream, culminating

T. brucei Chromosome 4 (0.98-1.47Mbp)

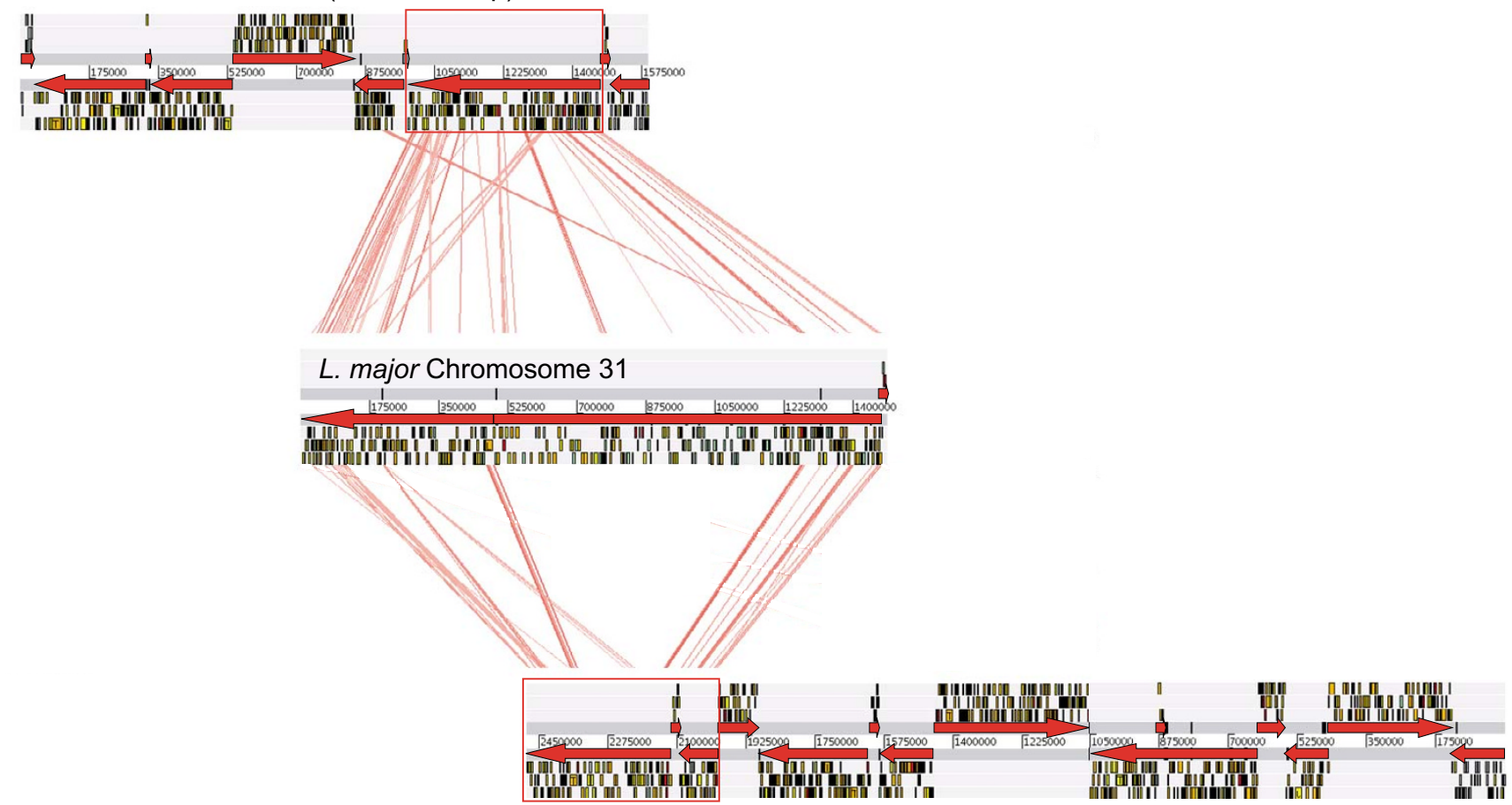

T. brucei Chromosome 8 (2.00-2.48Mbp)

Figure I

ACT comparison of whole Chromosomes 4 and 8 in T. brucei and Chromosome $3 \mathrm{I}$ in L. major. Chromosomes are presented as in GeneDB, with both forward and reverse strands and loci represented as coloured rectangles; grey arrows indicate the direction of transcription along polycistronic regions. Red rectangles denote the boundaries of the duplicated region on each chromosome; significant tBLASTx matches between homologous genes are linked by red coloured lines. Note that, while indicative, not all sequence affinity between chromosomes was detected in this tBLASTx search. 
in several genes that include a receptor-type adenylate cyclase (74: Tb927.4.3860). This is preceded upstream by 10 loci that were not conserved on Chromosome 4, and then a strand-switch region. Hence, each duplicon was bound by strand-switch regions and approximately 0.5 $\mathrm{Mb}$ in size, and gene order between the two regions was colinear and anti-parallel.

The location of homologs of the duplicated genes in $T$. cruzi and L. major genome sequences showed that gene order was largely conserved in these species. This served two purposes; first, it showed that both duplicons corresponded to the entirety of Chromosome 31 in L. major, although, as Figure 1 shows, this chromosome is larger and contains other genes besides those retained in T. brucei. Complete chromosomal structure is not established in T. cruzi. And second, it enabled the ancestral gene order of the pre-duplication chromosome to be inferred, through the classification of duplicated genes as shared (i.e. present on both duplicons and in an outgroup), lost (i.e. present on one duplicon and in an outgroup) or gained (not present in an outgroup). 74 loci were shared by both duplicons and other species. 57 genes on Chromosome 4 were absent from Chromosome 8 but present in other species, indicating that they were lost. Similarly, 27 genes on Chromosome 8 and present in other species were absent from Chromosome 4 . Therefore, $47 \%$ of all duplicated loci were retained as paralogs on both duplicons. Furthermore, 7 and 18 loci were present on Chromosomes 4 and 8 respectively, but absent from other species, suggesting that they were independently gained postduplication. A detailed and interactive figure showing the colinear gene order of the duplicons, and with links to the T. brucei genome sequence, is available from the GeneDB website [49], (also included here as Additional data file 1 ). Shared and lost genes appeared to differ in the presence of transmembrane helices (TMH) and putative signal peptides. A two-sample t-test assuming heteroscedasticity confirmed that conserved paralogs included significantly more TMH $(\mu=1.45, \mathrm{df}=102, \mathrm{p}=0.011)$ and signal peptides $(\mu=0.2, \mathrm{df}=134, \mathrm{p}=0.033)$ than singleton genes.

It was concluded from comparison of T. brucei and T. congolense genome sequences that the segmental duplication is restricted to T. brucei. It was initially observed that the preliminary assembly for chromosomes 4 and 8 in T. congolense did include homoeologous regions to the T. brucei duplicons. The putative duplicons in T. congolense showed conserved synteny and numerous retained paralogs, as in T. brucei. However, comparison of sequence divergence between paralogs showed that the preliminary T. congolense genome sequence did not contain an ortholog for each paralog seen in T. brucei, as expected if the duplication had occurred prior to speciation. Of 42 instances where a locus had been duplicated and retained on both duplicons, by both species, the $T$. congolense 'paralogs' were identical in nucleotide sequence in every case; furthermore, the intergenic sequences of 'paralogous' regions were also identical. Further examination of the sequence reads for $T$. congolense homologs identified putative alleles, but nothing to suggest the presence of orthologs to both $T$. brucei duplicates. It is implausible that while $T$. brucei paralogs have diverged considerably in most cases, and intergenic regions have little or no affinity, the corresponding regions in $T$. congolense should have remained entirely unchanged over the same time-span. With the completion of the $T$. congolense genome, it will hopefully become clear why preliminary assemblies reproduced the structure of the T. brucei genome sequence; but $T$. congolense certainly does not display the evolutionary dynamics seen in T. brucei and does not share in the effects of the duplication event (in terms of the derivation of novel genes).

\section{(b) Sequence divergence of conserved paralogs}

The remaining analyses dealt with the consequences of segmental duplication for the divergence of conserved paralogs present on both duplicons. There was considerable variation in the sequence identity between paralogs, as shown in Additional data file 1 and recorded in Additional data file 2. There were instances of extreme conservation between paralogs, for example, a myosin heavy chain kinase A showing 98\% identity (26: Tb927.4.4970 and $\mathrm{Tb} 927.8 .7450$ ), and of extreme divergence, for example, a monoglyceride lipase showing $39 \%$ identity (53: Tb927.4.4360 and Tb927.8.8020). Other paralogous, hypothetical genes showed as little as $5 \%$ identity (e.g., 7 : Tb927.4.5330 and Tb927.8.7060). Figure 2 shows a bimodal frequency distribution of nucleotide sequence identity for all shared paralogs. Coding sequences either changed little, retaining $70-100 \%$ identity, or diverged to around $40 \%$ identity; but there were low numbers of CDS with identity at $50-60 \%$, or less than $30 \%$.

Sequence divergence among NCS was generally greater and bimodality was more pronounced. There were paralogs with almost identical 5' and 3' untranscribed regions (UTR), for instance, paralogous RNA polymerase IIA large subunits (22: Tb927.4.5020 and Tb927.8.7400) had identical 3' UTRs over 400 bp; but highly divergent, indeed unalignable, UTRs were a more typical observation. Figure 2 identifies many NCS with less than $25 \%$ identity, which is no greater than expected by chance. These cases are largely an expression of comparisons between unaligned sequences; however, in some instances a part of the CDS or NCS aligned well but constituted only a minor fraction of the whole alignment, producing a value below 0.25 . This explains how paralogy was established between sequences with less than 0.25 identity. The bimodality of sequence divergence is further illustrated in Figure 3 


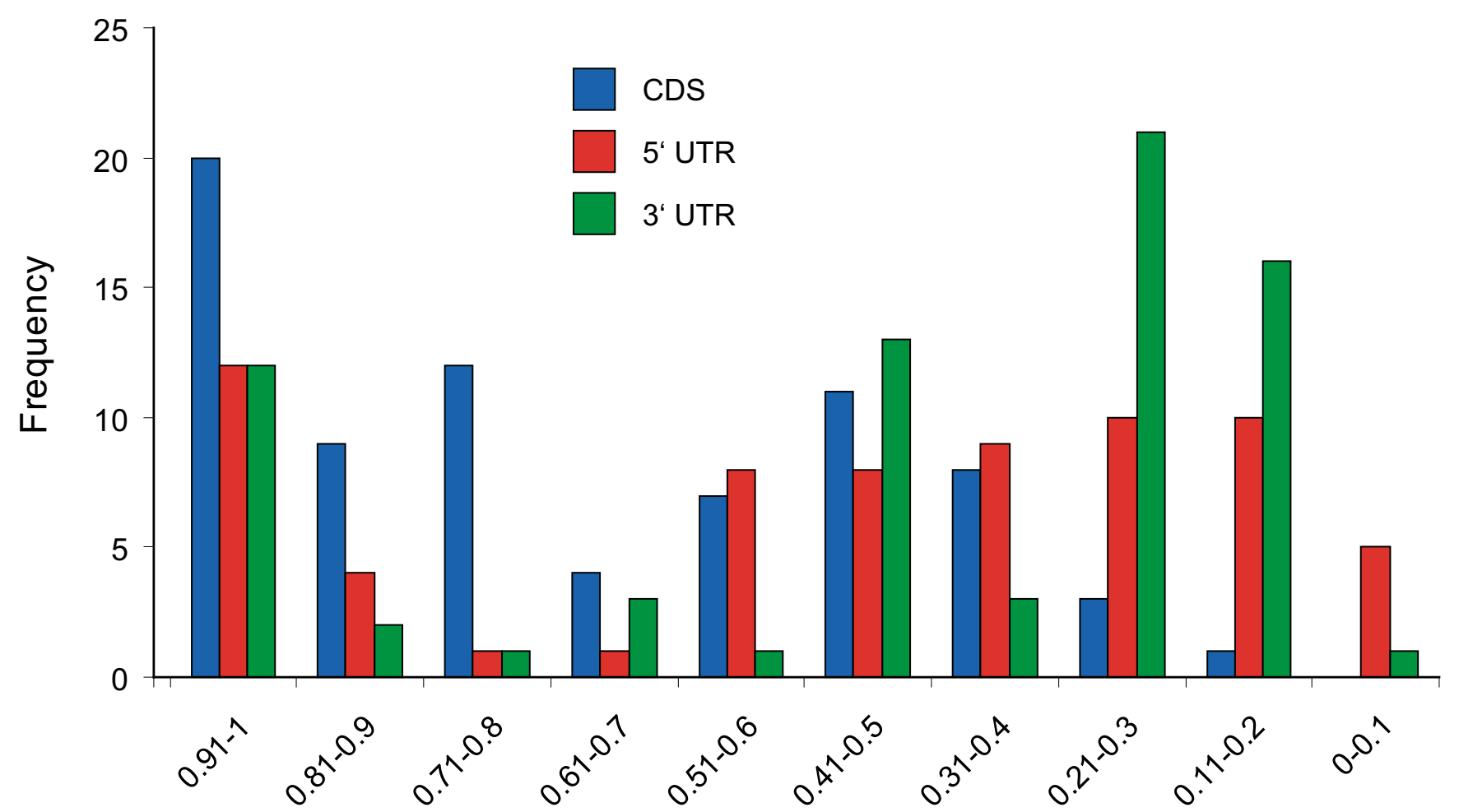

\section{Sequence identity}

\section{Figure 2}

Frequency distribution of sequence identity between paralogs retained on both duplicons. Nucleotide sequence identity is recorded for coding regions and each UTR.

a) 5' UTR

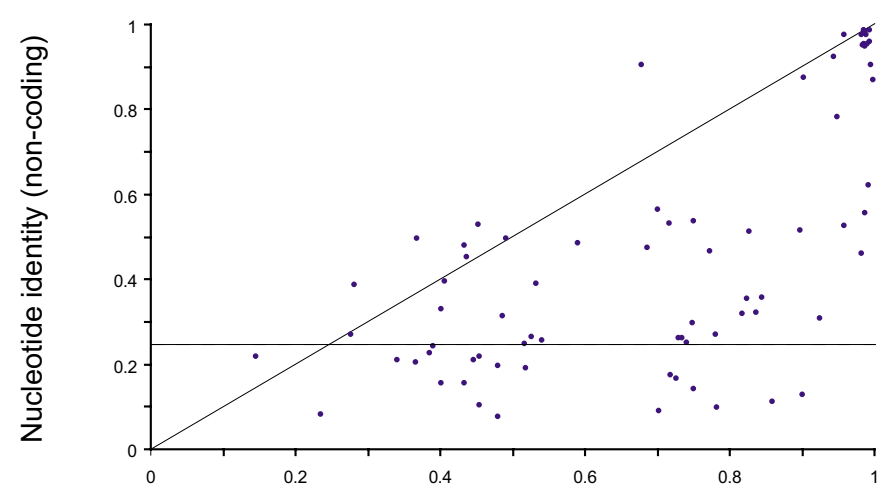

b) 3' UTR

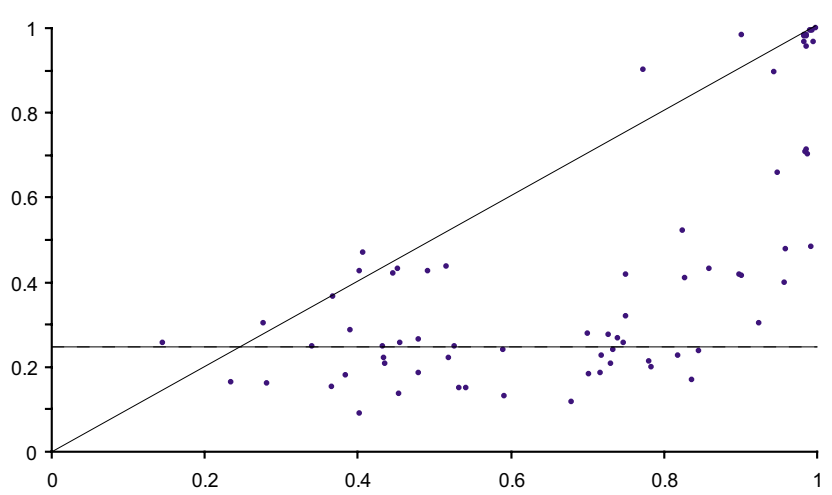

Amino acid identity (coding region)

Figure 3

Sequence divergence between paralogs. Values for coding regions are correlated with 5' UTR (a) and 3'UTR (b) regions. Horizontal dashed lines denote the $25 \%$ nucleotide identity expected between two unrelated sequences. 
where coding and non-coding identities for each paralogous gene pair are correlated. Both panels A and B, though especially the 3' UTR comparison, clearly show gene pairs consistent with a 1:1 relationship, including both conserved and divergent cases. However, in both cases there was a deficit of gene pairs falling in the 0.6-0.8 range and a surfeit of those falling into the bottom-right quadrant of the graph. These cases have CDS identities between 0.6 and 0.8 , but with much lower values for NCS. Once again, many NCS values fell below 0.25, reflecting those sequences that were unaligned. In summary, despite being formed at the same time, divergence of duplicated coding and non-coding sequences varied widely along the duplicons. The bimodality of divergence values indicated that fewer duplicate sequences had diverged moderately than expected; rather duplicates had remained largely unchanged or become very different.

\section{(c) Evolutionary rate asymmetry}

Relative rates tests were used to analyse paralogous CDSs for significant asymmetry in the rate of evolution postduplication; all results are shown in Additional data file 3. Canonical relative rates tests identified 12 cases where one paralog had evolved significantly faster; these are listed in Table 1, and indicated by blue arrows in Additional data file 1. Among these cases there were two gene pairs, 3methylcrotonyl-CoA carboxylase (5: Tb927.4.5350 and Tb927.8.6970) and dihydrolipoamide dehydrogenase (20: Tb927.4.5050 and Tb927.8.7380), where the rate asymmetry coincided with apparent psuedogenisation of one paralog. Elsewhere, monoglyceride lipase, noted above as having exceptional sequence divergence also displayed rate asymmetry (in favour of the Chromosome 8 copy), as did paralogous tandem gene arrays of glycosyltransferase (57: Tb927.4.4290 and Tb927.8.8090) and amino acid transporter genes (69: Tb927.4.4020 and Tb927.8.8220). The largest asymmetry in evolutionary rate occurred between paralogs of a mitotic centromerassociated kinesin (73: Tb927.4.3910 and Tb927.8.8350); the rate of non-synonymous substitutions per non-synonymous site for the Chromosome 4 lineage was 0.4386 , compared with 0.6062 for the Chromosome 8 lineage $\left(\mathrm{p}=1.00 \times 10^{-7}\right)$. The Bayesian relative rates test was more conservative, identifying 4 cases of significant asymmetry that are detailed in Table 2; each of these corroborated a significant result obtained using the canonical test. A complete account of the Bayesian relative rates tests is given in Additional data file 4.

\section{(d) Non-neutral evolution}

One factor capable of causing differential evolutionary divergence between paralogs is natural selection. For all genes along the duplicons, the action of selection on individual codons was examined using SLAC, FEL and REL algorithms to calculate $\omega$. The T. congolense homolog was used as an outgroup comparison in these tests. Additional file 5 shows the global $\omega$ values for all loci, none of which suggested net positive selection (i.e., $\omega>1$ ). Most singleton loci and paralogous genes were under negative, purifying selection when compared to their $T$. congolense homologs (i.e., $\omega<1$ ), which was reflected in a leftskewed frequency distribution of global $\omega$ and an average value of 0.219 ( \pm SD 0.099). Those paralogs showing extreme deficits in non-synonymous substitutions (i.e., $\omega$ $<0.1$ ) included an RNA polymerase IIA large subunit that was generally well conserved (22: Tb927.4.5020 and Tb927.8.7400), an ubiquinol-cytochrome $\mathrm{C}$ reductase (25: Tb927.4.4990 and Tb927.8.7430) and a translocating pyrophosphatase (51: Tb927.4.4380 and Tb927.8.7980), despite this being generally quite divergent. Although purifying selection was ubiquitous, FEL and REL tests detected 12 and 41 positively-selected codons respectively among both singleton loci and retained paralogs. However, the incidence of positive selection involving paralogous genes (and their outgroup) was significantly higher than for singletons and their outgroups, when combining the two tests $(\mathrm{p}=0.0125$, Fisher's exact test) or analysing them separately (FEL, p = 0.0059 ; REL, $\mathrm{p}=0.051$ ). Including an outgroup in these tests meant that some cases of positive selection could derive from the interspecific comparison, rather than the duplication event. Hence, when the outgroup was excluded, positive selection was detected in 21 duplicate loci; Table 3 describes the results for 16 loci that included positively-selected loci both with and without an outgroup comparison (these are also marked with red arrows in Additional file 1).

Beyond the ratio of amino acid replacements to silent substitutions, other patterns of sequence divergence could reflect non-neutral evolution. Paralogous sequences were scored for the ratio of 'invariable' to 'variable' mutations at non-synonymous and then synonymous sites. Significant disparity between these ratios was an indication of non-neutral evolution and was detected in 15 cases at the $\mathrm{p}=0.01$ level; these are shown in Table 4 and as green arrows on Additional data file 1. Details for all loci are shown in Additional data file 6. These comprised the tail in an over-dispersed distribution of G statistics, meaning that most cases showed negligible difference between substitution patterns at the distinct sites. Where significant disparity was observed, this was generally due to an excess of RI mutations, and there were four cases in particular where the number of invariable mutations outnumbered variable mutations at non-synonymous sites; these concerned three pairs of paralogous hypothetical genes: $\mathbf{6}$ (Tb927.4.5340 and Tb927.8.6980), 2 (Tb927.4.5380 and Tb927.8.6940), and 24 (Tb927.4.5000 and Tb927.8.7420); as well as paralogs of a single-copy amino acid transporter (40: Tb927.4.4730 and Tb927.8.7740). 
Table I: Shared, paralogous loci showing significant asymmetry in the rate of non-synonymous substitutions per site since duplication, as determined by the canonical relative rates test. Locus Identifer Chr4 Chr8

Description

CDS identity Interspecific CDS 5' UTR:

3' UTR:

RRT:

Identity: Identity Length (bp) Identity Length (bp) \# Sites $D_{n}$

$\Delta D_{n} \quad S D$ ratio $\quad P$

Chr4 Chr8

Chr4 Chr8

$5 \quad$ Tb927.4.5350 $\quad$ Tb927.8.6970 $\quad$ 3-methylcrotonyl-CoA carboxylase

0.983

0.661

0.652

0.976

42

0.984

327

$\begin{array}{lllllll}1361.5 & 0.253 & 0.241 & -0.012 & 0.004 & -2.797 & 0.005\end{array}$

$9 \quad$ Tb927.4.5310 Tb927.8.7110 S/T-protein kinase A

20 Tb927.4.5050 Tb927.8.7380 dihydrolipoamide dehydrogenase

$43 \quad$ Tb927.4.4550 Tb927.8.7780 Tb927.8.7760

$\begin{array}{llll}46 & \text { Tb927.4.4520 } & \text { Tb } 927.8 .7820 & \text { Tb927.8.7800 }\end{array}$

$\begin{array}{llll}51 & \text { Tb927.4.4350 } & \text { Tb927.8.8030 } & \text { Tb927.8.7950 }\end{array}$

54 Tb927.4.4380 Tb927.8.7980 monoglyceride lipase

$\begin{array}{llll}58 & \text { Tb927.4.4240 } & \text { Tb927.8.8070 glycosyltransferase }\end{array}$

$59 \quad$ Tb927.4.4220 Tb927.8.8140 Tb927.8.8070/8110

$\begin{array}{lll}70 & \text { Tb927.4.3970 Tb927.8.8320 amino acid transporter }\end{array}$

$\begin{array}{lll}0.438 & 0.416 & 0.192\end{array}$

$\quad-\quad-\quad 0.261$

0.199

0

0.222

18

73 Tb927.4.3910 Tb927.8.8350 Tb927.4.3920 (TMH) 
Table 2: Shared, paralogous loci showing significant asymmetry in total substitution rate since duplication $(P<0.05)$, as determined by the Bayesian relative rates test.

\begin{tabular}{|c|c|c|c|c|c|c|c|c|c|c|}
\hline \multirow[t]{2}{*}{ Locus } & \multirow{2}{*}{$\begin{array}{l}\text { Identifier } \\
\text { Chr4 }\end{array}$} & \multirow[b]{2}{*}{ Chr8 } & \multicolumn{2}{|c|}{ Chromosome 4: } & \multirow[b]{2}{*}{ Range } & & \multicolumn{2}{|c|}{ Chromosome 8: } & \multirow[b]{2}{*}{ Range } & \\
\hline & & & Average & SD & & & Average & SD & & \\
\hline 42 & Tb927.4.4570 & Tb927.8.7760 & 0.4483 & 0.0220 & 0.404 & 0.492 & 0.577 I & 0.0265 & 0.524 & 0.630 \\
\hline 45 & Tb927.4.4530 & Tb927.8.7800 & 0.4914 & 0.0284 & 0.435 & 0.548 & 0.3339 & 0.0240 & 0.286 & 0.382 \\
\hline 50 & Tb927.4.4400 & Tb927.8.7950 & 0.6422 & 0.0269 & 0.588 & 0.696 & 0.5099 & 0.0232 & 0.464 & 0.556 \\
\hline 58 & Tb927.4.4240 & Tb927.8.8070 & 0.4534 & 0.0774 & 0.299 & 0.608 & $0.84 I 4$ & 0.1162 & 0.609 & 1.074 \\
\hline
\end{tabular}

Locus number refers to Additional file I.

Values in bold denote the paralogs with the faster evolutionary rate.

\section{(e) Taxonomic distribution PCR assay}

Three locations were selected where a shared paralog on both chromosomes was followed downstream by different, single-copy genes on each duplicon. For each location, a genome containing both duplicons should yield two distinct PCR products. The presence of both duplicons in other T. brucei strains would therefore be demonstrated by amplification of all six PCR products of the correct size. Additional data file 7 shows that this was observed in the subspecies to which the original genome sequence belongs (T. b. brucei), but also in T. b. gambiense, $T$. $b$. rhodiense and T. evansi, confirming that the duplication was common to all members of the T. brucei clade.

\section{Discussion}

The duplication of a chromosome-sized region of the $T$. brucei genome was identified, based on the colinear gene order along $0.5 \mathrm{MB}$ regions of chromosomes 4 and 8 . Comparisons with homoeologous regions in other trypanosomatids confirmed that the duplicated region corresponded to the entire chromosome 31 in Leishmania spp. and was unique to T. brucei. In addressing the aims of this study, it has been shown that although substantial gene loss occurred after duplication, $47 \%$ of all duplicated loci had been retained as conserved paralogs. The functions of retained compared with deleted genes suggested that gene loss was selective. Divergence of conserved paralogs was bimodal, particularly in the case of NCSs; UTRs either remained highly conserved or were radically remodelled. Sequence divergence was also characterised by ubiquitous purifying selection, frequent rate asymmetry between paralogs and occasional positive selection, which nonetheless occurred significantly more often among duplicates than single-copy genes. So taken together, the patterns of observed structural change suggested that at least some conserved paralogs were functionally innovative.

\section{(a) Post-duplication effects on gene content}

Duplication events of this kind have not previously been recorded in trypanosomatid genomes. Karyotypic fluctuations appear to be reasonably frequent among trypano- somatids; in T. brucei the infrequent nature of reductive cell division produces triploid hybrid strains [50,51]; the irregularity of genetic exchange in these organisms also seems to cause widespread variation in ploidy in T. cruzi [7]. Among Trypanosoma spp. and Leishmania spp. fluctuations in repetitive, telomeric regions causes substantial variation in chromosome size $[4,5]$. However, the effect of these events seems to be restricted to karyotype, and has not had permanent effects on genetic complement. The duplication event recorded here differs in nature because its effects on genetic complement go beyond spatio-temporal fluctuations in copy number; the duplicons evolved through deletion of many genes, gain of a few others, and widespread divergence of retained paralogs, to create a novel and permanent addition to the T. brucei genome. Such expansions in genetic complement through large duplication events have emerged as primary evolutionary catalysts from various taxa; both yeasts [18-20] and angiosperms [14-17] are known to be palaeopolyploids, while the possibility of successive whole-genome duplications in vertebrates continues to be debated [24,51-55].

Comparisons of completed genomes from these organisms are illuminating the mechanisms of large duplication events. Koszul et al. (2003) [56] examined reversion to wild-type in Saccharomyces cerevisiae after enforcing a growth defect; the majority of revertant strains resulted from spontaneous duplication events, ranging in size between 41 and $655 \mathrm{~kb}$. This and other observations [57] suggest that large duplication events result from damage incurred during DNA replication and its subsequent repair. The locations of such breakpoints in yeast also support the view that damage occurs at specific points of weakness, for instance termination sites, repetitive regions and those containing mobile elements $[58,59]$. Similarly, in $T$. brucei, the upstream junction of the chromosome 4 duplicon comprised a $40 \mathrm{~kb}$ region with almost no open reading frames but several mini-satellite loci and both DIRE and RIME mobile elements.

Large duplication events make immediate additions to genomic repertoire, many of which will prove lasting. 
Table 3: Retained paralogs with codons showing evidence for positive or negative selection.

\begin{tabular}{|c|c|c|c|c|c|c|c|c|c|c|}
\hline \multirow[t]{2}{*}{ Locus } & \multirow[t]{2}{*}{ Identifier Chr4 } & \multirow[t]{2}{*}{ Chr8 } & \multicolumn{2}{|c|}{ Selection analysis: With outgroup } & \multirow[b]{2}{*}{+} & \multirow[b]{2}{*}{-} & \multirow[b]{2}{*}{+ codons } & \multicolumn{2}{|c|}{ Without outgroup } & \multirow[b]{2}{*}{ - codons } \\
\hline & & & Method & Global $\omega$ & & & & - codons & + codons & \\
\hline \multirow[t]{3}{*}{6} & Tb927.4.5340 & Tb927.8.6980 & SLAC & 0.382 & 0.369 & 0.462 & 0 & 0 & & \\
\hline & & & FEL & & & & 0 & 54 & & \\
\hline & & & REL & & & & 2 & 65 & 67 & 0 \\
\hline \multirow[t]{3}{*}{8} & Tb927.4.5320 & Tb927.8.7090 & SLAC & 0.333 & 0.325 & 0.418 & 0 & 0 & & \\
\hline & & & FEL & & & & 0 & 84 & & \\
\hline & & & REL & & & & 5 & 340 & 7 & 6 \\
\hline \multirow[t]{3}{*}{10} & Tb927.4.5300 & Tb927.8.7I40 & SLAC & 0.289 & 0.271 & 0.364 & 0 & 1 & & \\
\hline & & & FEL & & & & 1 & 44 & & \\
\hline & & & REL & & & & 15 & 192 & 20 & 7 \\
\hline \multirow[t]{3}{*}{12} & Tb927.4.5220 & Tb927.8.7190 & SLAC & 0.315 & 0.302 & 0.415 & 0 & 0 & & \\
\hline & & & FEL & & & & 0 & 31 & & \\
\hline & & & REL & & & & 6 & 77 & 19 & 7 \\
\hline \multirow[t]{3}{*}{14} & Tb927.4.5।80 & Tb927.8.7220 & SLAC & 0.201 & 0.192 & 0.266 & 0 & 0 & & \\
\hline & & & FEL & & & & 0 & 73 & & \\
\hline & & & REL & & & & 4 & 135 & 2 & 49 \\
\hline \multirow[t]{3}{*}{21} & Tb927.4.5030 & Tb927.8.7390 & SLAC & 0.072 & 0.062 & 0.112 & 0 & 0 & & \\
\hline & & & FEL & & & & 0 & 65 & & \\
\hline & & & REL & & & & 5 & 286 & 3 & 24 \\
\hline \multirow[t]{3}{*}{27} & Tb927.4.4960 & Tb927.8.7460 & SLAC & 0.188 & 0.176 & 0.246 & 0 & 0 & & \\
\hline & & & FEL & & & & 0 & 76 & & \\
\hline & & & REL & & & & 7 & 289 & 49 & 0 \\
\hline \multirow[t]{3}{*}{31} & Tb927.4.4920 & Tb927.8.7500 & SLAC & 0.413 & 0.363 & 0.508 & 0 & 0 & & \\
\hline & & & FEL & & & & 0 & 13 & & \\
\hline & & & REL & & & & 6 & 63 & 15 & 0 \\
\hline \multirow[t]{3}{*}{40} & Tb927.4.4730 & Tb927.8.7740 & SLAC & 0.148 & 0.138 & 0.202 & 0 & 1 & & \\
\hline & & & FEL & & & & 0 & 45 & & \\
\hline & & & REL & & & & 4 & 201 & 40 & 0 \\
\hline \multirow[t]{3}{*}{43} & Tb927.4.4550 & Tb927.8.7780 & SLAC & 0.199 & 0.193 & 0.261 & 0 & 1 & & \\
\hline & & & FEL & & & & 0 & 158 & & \\
\hline & & & REL & & & & 11 & 17 & 5 & 7 \\
\hline 46 & Tb927.4.4520 & Tb927.8.7820 & SLAC & 0.342 & 0.33 & 0.426 & 0 & 0 & & \\
\hline & & & FEL & & & & I & 57 & & \\
\hline & & & REL & & & & i & 73 & 6 & 0 \\
\hline 49 & Tb927.4.4470 & Tb927.8.7860 & SLAC & 0.256 & 0.253 & 0.335 & 0 & 2 & & \\
\hline & & & FEL & & & & 0 & 214 & & \\
\hline & & & REL & & & & 2 & 187 & 20 & 0 \\
\hline 50 & Tb927.4.4400 & Tb927.8.7950 & SLAC & 0.369 & 0.365 & 0.447 & 0 & 10 & & \\
\hline & & & FEL & & & & 4 & 191 & & \\
\hline & & & REL & & & & 0 & 121 & 2 & 0 \\
\hline 52 & Tь927.4.4370 & Tb927.8.8000 & SLAC & 0.216 & 0.207 & 0.28 & 0 & 0 & & \\
\hline & & & FEL & & & & 0 & 91 & & \\
\hline & & & REL & & & & 49 & 68 & 8 & 0 \\
\hline 62 & Tb927.4.4I60 & Tb927.8.8170 & SLAC & 0.282 & 0.273 & 0.345 & 0 & 1 & & \\
\hline & & & FEL & & & & I & 94 & & \\
\hline & & & REL & & & & 3 & $|8|$ & 5 & 0 \\
\hline 71 & Tb927.4.3950 & Tb927.8.8330 & SLAC & 0.249 & 0.242 & 0.318 & 0 & 0 & & \\
\hline & & & FEL & & & & 1 & 115 & & \\
\hline & & & REL & & & & 14 & 458 & 27 & 41 \\
\hline
\end{tabular}

However, the conclusion from several model organisms is that such events are typically followed by substantial gene loss [11-18], often resulting in 'diploidisation' and the restoration of original gene number [22]. Unusually, almost half of duplicated loci recorded here are retained as paralogs; this contrasts with $28.6 \%$ and just $~ 20 \%$ following whole-genome duplications in A. thaliana [60] and Oryza sativa [29] respectively, and perhaps $10 \%$ in yeast [61]. Such recognised WGD events are shared across species or genera, and are undoubtedly ancient. By contrast, the present case is apparently restricted to T. bruce $i$ and is certainly absent from other principal trypanosome clades. Its 
Table 4: Shared, paralogous loci showing significant disparity $(p<0.005)$ between the ratio of invariable to variable mutations at synonymous (S) and non-synonymous sites $(R)$ respectively, as determined by $G$ test.

\begin{tabular}{|c|c|c|c|c|c|c|c|c|c|}
\hline \multirow[t]{2}{*}{ Locus } & \multirow[t]{2}{*}{ Description } & \multicolumn{4}{|c|}{ Mutation type: } & \multirow[t]{2}{*}{ G } & \multirow[t]{2}{*}{ Ratio $_{R}$} & \multirow[t]{2}{*}{ Ratio $_{S}$} & \multirow[t]{2}{*}{ Ratio $_{\text {RS }}$} \\
\hline & & RI & RV & SI & SV & & & & \\
\hline 2 & Tb927.4.5380 (alcohol dehydrogenase-like) & 436 & 10 & 310 & 21 & 8.155 & 43.60 & 14.76 & 2.954 \\
\hline 6 & Tb927.4.5340 & 356 & 284 & 147 & 191 & 13.03 & 1.254 & 0.770 & 1.629 \\
\hline 8 & Ть927.4.5320 & 197 & 384 & 105 & 314 & 9.131 & 0.513 & 0.334 & 1.534 \\
\hline 24 & Tb927.8.7420 & 256 & 5 & 210 & 15 & 6.937 & 51.20 & 14.00 & 3.657 \\
\hline 29 & Tb927.8.7480 & 135 & 484 & 53 & 317 & 8.674 & 0.279 & 0.167 & 1.668 \\
\hline 35 & Tb927.8.7580 (TMH/SP) & 201 & 539 & 75 & 400 & 22.05 & 0.373 & 0.188 & 1.989 \\
\hline 36 & amino acid transporter & 132 & 279 & 89 & 379 & 19.92 & 0.473 & 0.235 & 2.015 \\
\hline 37 & amino acid transporter & 54 & 191 & 41 & 287 & 9.081 & 0.283 & 0.143 & 1.979 \\
\hline 41 & amino acid transporter & 139 & 60 & 83 & 77 & 12.09 & 2.317 & 1.078 & 2.149 \\
\hline 49 & Tb927.8.7850 & 210 & 598 & 84 & 429 & 17.25 & 0.351 & 0.196 & 1.793 \\
\hline 50 & adenylate cyclase GRESAG 4 & 73 & 502 & 98 & 414 & 8.458 & 0.145 & 0.237 & 0.614 \\
\hline 51 & Tb927.8.7950 & 430 & 1671 & 178 & 905 & 7.649 & 0.257 & 0.197 & 1.308 \\
\hline 58 & UDP-GIcNAc-dependent glycosyltransferase & 67 & 318 & 24 & 247 & 10.12 & 0.211 & 0.097 & 2.168 \\
\hline 62 & Tb927.4.4I80 & 234 & 775 & 83 & 475 & 15.94 & 0.302 & 0.175 & 1.728 \\
\hline 68 & Tb927.8.8270 & 263 & 748 & 97 & 485 & 19.05 & 0.352 & 0.200 & 1.758 \\
\hline
\end{tabular}

cladistic distribution suggests that is a relatively recent event, which may explain the large fraction of genes retained in comparison with other large duplication events. The fate of gene duplicates is complex and depends both on taxon [27] and function, incorporating protein complexity [62], dose sensitivity and 'connectivity' $[60,63,64]$. Some observations suggest that genes encoding simpler products, with fewer interactions with regulators or targets around the cell (i.e., lower 'connectivity'), are retained in duplicate more often. Hence, in yeast complex proteins are retained less often as the number of subunits increases [65], subunits of heterodimers are less duplicable than those in homodimers [62] and there was a negative relationship between 'connectivity' and retention after duplication [66]. The 'balance' hypothesis explains such biases in terms of dose sensitivity [63]; effective gene expression depends on a dynamic equilibrium of regulatory factors, which is perturbed by unilateral duplications of regulatory genes or individual components of larger assemblages. Hence, genes that are more peripheral to the regulatory environment and dose-insensitive, such as surface-expressed genes, may be retained more often [66].

However, most analyses identifying selective gene loss report enrichment of highly connected and expressed genes, integral to cell function, for instance those associated with regulation, signal transduction, transcriptional control and protein-protein interactions [26,27]; this can apparently result in co-localisation of regulator and target loci over time $[60,67]$. Yet, these studies have addressed WGD events and the dosage balance hypothesis accommodates them if gene loss is considered scale-dependent [68]. Dosage balance can be preserved either by retaining all components of a regulatory network, where a duplica- tion is large enough to include a gene plus all its interacting loci, or by deleting them, where the event has been smaller. In T. brucei, retained paralogs were enriched with TMH and signal peptides, showing that gene loss was selective after duplication, resulting in preferential retention of surface expressed genes, (e.g., amino acid transporters, adenylate cyclases, glycosyltransferases). Although the large number of uncharacterised genes limits our ability to scrutinise gene loss by function, metabolic enzymes such as metallopeptidases, components of the electron transfer chain, and various loci associated with the RNA synthesis and modification featured prominently among deleted duplicates. This is consistent with the preservation of dosage balance through deletion of loci with high connectivity, and retention of dose-insensitive proteins on the cell surface. Indeed, the process of removing dose-sensitive genes may be continuing through pseudogenisation of duplicates such as a dihydrolipoamide dehydrogenase (20: Tb927.8.7380). Prior to duplication, this locus was duplicated in tandem; after duplication, one copy was lost from chromosome 8 and another is currently being deleted from chromosome 4, thereby restoring the original copy number.

\section{(b) Post-duplication effects on gene sequence}

For those gene duplicates that are preserved, duplication marks the beginning of genetic divergence. Paralogs will diverge over time, unless gene conversion homogenises their sequences at a much faster rate [46]. The speed and magnitude of evolutionary change depends on the selective environment following duplication, the nature of which is likely to vary by case. The role of adaptive evolution in neofunctionalisation and subfunctionalisation models differs, but setting positive selection aside, the common theoretical expectations are that evolutionary 
rate should accelerate in one or both duplicates as functional change accumulates. Regulatory regions should also be remodelled, either to effect functional change (i.e., under a DDC model) or to preserve it. In this case, most features of sequence divergence - the prevalence of negative selection, rate asymmetry, and excessive numbers of non-synonymous substitutions - indicate that post-duplication sequence change has been functional. At a basic level, patterns of coding and non-coding sequence divergence displayed a deficit in moderate divergence, indicating that regulatory regions in particular remained conserved or were entirely remodelled. Therefore, given that paralogs could be conservative or innovative with respect to both CDSs and NCSs, it follows that four types of dynamics were observed, examples of which are listed in Table 5.

Table 5: Sequence divergence of shared, paralogous loci, displaying four different evolutionary dynamics.

\begin{tabular}{|c|c|c|c|c|c|}
\hline \multirow[t]{2}{*}{ Locus } & \multicolumn{2}{|l|}{ Identifier } & \multirow[t]{2}{*}{ Annotation } & \multicolumn{2}{|c|}{ Sequence identity: } \\
\hline & Chr4 & Chr8 & & CDS & 3'UTR \\
\hline \multicolumn{6}{|c|}{ Conserved CDS/Conserved NCS } \\
\hline I & Tb927.4.5390 & Tb927.8.6930 & serine/threonine-protein kinase NrkA & 0.983 & 0.968 \\
\hline 2 & Tb927.4.5380 & Tb927.8.6940 & quinonprotein alcohol dehydrogenase-like & $0.993^{a}$ & 0.995 \\
\hline 3 & Tb927.4.5370 & Tb927.8.6950 & dynein light chain $2 B$ & 0.994 & 0.994 \\
\hline 4 & Tb927.4.5360 & Tb927.8.6960 & $\mathrm{TMH} / \mathrm{SP}$ & 0.987 & 0.984 \\
\hline 5 & Tb927.4.5350 & Tb927.8.6970 & 3-methylcrotonyl-CoA carboxylase & $0.983^{b}$ & 0.984 \\
\hline 22 & Tb927.4.5020 & Tb927.8.7400 & RNA polymerase IIA largest subunit & 0.999 & $\mathrm{l}$ \\
\hline 24 & Tb927.4.5000 & Tb927.8.7420 & C2 calcium/lipid-binding region, CaLB & $0.995^{a}$ & 0.966 \\
\hline 30 & Tb927.4.4930 & Tb927.8.7490 & & 0.987 & 0.956 \\
\hline 64 & Tb927.4.4I50 & Tb927.8.8180 & & 0.991 & 0.995 \\
\hline 65 & Tb927.4.4I40 & Tb927.8.8190 & & 0.986 & 0.981 \\
\hline 67 & Tb927.4.4I20 & Tb927.8.8210 & & 0.984 & 0.982 \\
\hline \multicolumn{6}{|c|}{ Divergent CDS/Divergent NCS } \\
\hline 7 & Tb927.4.5330 & Tb927.8.7060 & EGF/Laminin domain & 0.235 & 0.165 \\
\hline 10 & Tb927.4.5240 & Tb927.8.7I40 & UDP-GIcNAc-dependent glycosyltransferase & 0.455 & 0.258 \\
\hline 11 & Tb927.4.5230 & Tb927.8.7180 & & 0.385 & 0.181 \\
\hline 12 & Tb927.4.5220 & Tb927.8.7190 & $\mathrm{SP}$ & 0.402 & 0.09 \\
\hline 18 & Tb927.4.5। 20 & Tb927.8.7260 & kinetoplast-associated protein & 0.368 & 0.365 \\
\hline 29 & Tb927.4.4940 & Tb927.8.7480 & phosphopantetheine attachment site & $\left.0.49\right|^{a}$ & 0.427 \\
\hline 33 & Tb927.4.4900 & Tb927.8.7550 & & 0.282 & 0.16 \\
\hline 34 & Tb927.4.4890 & Tb927.8.7560 & $\mathrm{TMH}$ & 0.454 & 0.137 \\
\hline 35 & Tb927.4.4880 & Tb927.8.7580 & TMH/SP, Zn-finger protein & $0.48^{a}$ & 0.264 \\
\hline 38 & Tb927.4.4810 & Tb927.8.7710 & $\mathrm{TMH}$ & 0.402 & 0.425 \\
\hline 39 & Tb927.4.4790 & Tb927.8.7720 & $\mathrm{TMH} / \mathrm{SP}$ & 0.391 & 0.288 \\
\hline 42 & Tb927.4.4580 & Tb927.8.7750 & protein kinase & 0.446 & 0.422 \\
\hline 46 & Tb927.4.4530 & Tb927.8.7800 & SPla/RYanodine receptor SPRY & $0.407^{b}$ & 0.47 \\
\hline 47 & Tb927.4.4520 & Tb927.8.7820 & cold-shock protein, DNA-binding & 0.434 & 0.222 \\
\hline 48 & Tb927.4.4500 & Tb927.8.7830 & & 0.34 & 0.25 \\
\hline 51 & Tb927.4.4400 & Tb927.8.7950 & leucine rich repeat & $0.433^{\mathrm{ab}}$ & 0.25 \\
\hline 57 & Tb927.4.4310 & Tb927.8.8050 & spectrin repeat & 0.366 & 0.152 \\
\hline 59 & Tb927.4.4240 & Tb927.8.8070 & Zinc finger, $\mathrm{C} 2 \mathrm{H} 2$-type & 0.145 & 0.258 \\
\hline 60 & Tb927.4.4220 & Tb927.8.8I40 & small GTP-binding rab protein & 0.452 & 0.433 \\
\hline 62 & Tb927.4.4I80 & Tb927.8.8160 & & $0.48^{a}$ & 0.186 \\
\hline 69 & Tb927.4.4040 & Tb927.8.8280 & & 0.436 & 0.209 \\
\hline 71 & Tb927.4.3970 & Tb927.8.8320 & & 0.277 & 0.302 \\
\hline \multicolumn{6}{|c|}{ Conserved CDS/Divergent NCS } \\
\hline 9 & Tb927.4.5310 & Tb927.8.7II0 & serine/threonine-protein kinase $\mathrm{A}$ & $0.845^{b}$ & 0.239 \\
\hline 15 & Tb927.4.5I60 & Tb927.8.7240 & $\mathrm{TMH} / \mathrm{SP}$ & 0.837 & 0.169 \\
\hline 17 & Tb927.8.7250 & & & 0.958 & 0.4 \\
\hline 20 & Tb927.4.5050 & Tb927.8.7380 & dihydrolipoamide dehydrogenase & $0.924 b$ & 0.303 \\
\hline 45 & Tb927.4.4540 & Tb927.8.7790 & LSDI zinc finger & 0.818 & 0.227 \\
\hline 54 & Tb927.4.4360 & Tb927.8.8020 & monoglyceride lipase & $0.783^{b}$ & 0.199 \\
\hline 56 & Tb927.4.4330 & Tb927.8.8040 & diadenosine tetraphosphatase & 0.781 & 0.214 \\
\hline \multicolumn{6}{|c|}{ Divergent CDS/Conserved NCS } \\
\hline 63 & Tb927.4.4160 & Tb927.8.8I70 & SP, CheY-like domain & 0.773 & 0.901 \\
\hline 65 & Tb927.4.5I50 & Tb927.8.7240 & & 0.901 & 0.983 \\
\hline
\end{tabular}

a denotes evidence for 'constant but different' non-synonymous mutations.

b denotes evidence for significant rate asymmetry between retained paralogs. 
First, for some duplicates both CDS and NCS experienced few substitutions and remained structurally conserved. Such loci included RNA polymerase IIA (22), dynein light chain 2B (3), a 3-methylcrotonyl-CoA carboxylase (5), as well as many other hypothetical genes where divergence in either CDS or NCS did not exceed 5\%. These cases suggest that some genes involved in core cellular functions were retained to increase dose, and have been constrained by strong purifying selection. The substantial divergence of regulatory regions elsewhere makes a compelling argument for conservation of expression profile in these instances. However, high sequence identity does not preclude important structural changes, since two examples of this dynamic (24 and 2) also showed non-neutral substitution patterns, with significant excesses of 'constant-butdifferent' amino-acid replacements. This dynamic might also occur where gene conversion homogenises gene copies in trans, although no evidence was seen for this here.

The second dynamic involved substantial divergence in both CDS and NCS. These cases mostly involved hypothetical genes, which is intuitive since slowly-evolving genes fundamental to cellular function are more likely to be annotated; many possessed features indicative of surface-expression, and a GTP-binding rab protein (59) showing $45 \%$ and $43 \%$ divergence in CDS and NCS respectively was included. Since these paralogs often diverged beyond recognition in parts of their structures, such that functional conservation is implausible, they are the best candidates for neofunctionalisation. Positive selection was detected for individual codons at some loci with this dynamic; for example 10, 12, 46, 62 and 71. However, none of the paralogous sequences displayed a global $\omega$ above 1 , and positive selection was not conspicuously strong. Documented cases of neofunctionalisation are sparse, perhaps because it is easier to elucidate the functional differences between gene duplicates when sequence identity remains high. One instance, the evolution of antifreeze glycoproteins from trypsinogen-like proteases in Antarctic notothenioid fish [69], demonstrates how gene duplication can be followed by fundamental remodelling of primary structures, involving the loss of functional domains and very low sequence identities, of the magnitude recorded in T. brucei. At least two conserved paralogs showed remodelling of this type, and so are candidates for radical new functions. First, a kinetoplastid membrane protein (18: Tb927.4.5120 and Tb927.8.7260) was substantially shortened on chromosome 8 relative to chromosome 4 (and orthologs in other species), involving the deletion of a large repetitive Hint domain and a GPI anchor signal; these features suggest a membrane-bound signalling function that has been lost by the chromosome 8 paralog. Second, a hypothetical gene (72: Tb927.4.3920 and Tb927.8.8340) possessed a lipid-binding domain and 12 transmembrane helices, the latter were deleted from the chromosome 8 paralog, indicating that the protein had acquired a new position within the cell.

The third dynamic combined divergence of NCS with conservation of coding regions. This kind of change might indicate where regulation of a gene has evolved without much structural change, perhaps resulting in a novel expression profile. Genes in this category are mostly metabolic enzymes, e.g., protein kinase, lipase and phosphatase; these might be expected to evolve slowly with respect to primary structure, but they offer interesting avenues for further investigation since their regulatory regions have clearly been changed considerably. Several previous cases have shown that innovation can occur through changes to regulatory domains alone. Bhushan et al. (2005) [70] described paralogous metallopeptidases in A. thaliana with $75.6 \%$ protein identity but differential expression in tissue-specific manner. Segregation of the ancestral function between duplicates was achieved through regulatory change (although some structural change had also altered enzyme specificity), consistent with a subfunctionalisation model. Hua et al. (2003) [71] described a similar case in humans, where Nudt10 and Nudt11 were recently duplicated phosphohydrolases, the former expressed in liver, kidney and testis and the latter restricted to brain. However, these copies were identical at the protein level. Indeed, the evolution of proteins under strong structural constraints, such as Hox genes [72,73] through changes to cis-acting regulatory modules provides the most comprehensive evidence that subfunctionalisation via this mechanism is a general principle.

In the final dynamic coding regions diverged, while untranslated regions remained structurally conserved. Given that non-coding regions are typically less affected by negative selection, it is intuitive that this outcome was rare. Table 5 shows that in two cases non-coding regions were conserved ( $90 \%$ and $98 \%$ respectively), while CDS divergence was greater, but only considerably so for one hypothetical gene (63), likely to be involved in signal transduction. There was no evidence of significant rate asymmetry or non-neutral substitutions between these paralogous pairs, and so it seems more likely that strong selection to preserve regulatory regions was responsible for the dynamic. Therefore, these loci might represent odd examples of structural derivation in the absence of changes to expression profiles.

In summary, the diverse divergence rates observed, (despite the time of separation being constant), the instances of contrary divergence patterns in CDS and NCS, as well as significantly asymmetric change or adaptive substitutions among many paralogous pairs, all sug- 
gest that the duplication event had equally diverse functional consequences.

\section{Conclusion}

The T. brucei clade has been affected by the duplication and transposition of a large chromosomal block, perhaps due to a mistake during cell division. For events of this type, a surprisingly large proportion of gene duplicates were subsequently retained, including gene families known to be important at the host-parasite interface. Gene loss was selective, since surface-expressed genes were over-represented among conserved paralogs; this is consistent with a dosage balance hypothesis in which genes with low 'connectivity' within the cell are more likely to be preserved after segmental duplications because they are dose-insensitive. Sequence divergence of conserved paralogs followed several different evolutionary trajectories, sometimes accompanied by significant asymmetry in substitution rate and significant excesses of amino acid replacements, and generally more prone to adaptive evolution than singleton loci. Indeed, the structural change among coding and regulatory regions of conserved paralogs was often radical, providing strong indications that many of these cases involved functional change. The functional consequences of this duplication will become clear as hypothetical genes are annotated and the biological differences between paralogs investigated. However, by demonstrating considerable gene retention and structural divergence, this study has established that the duplication made a significant contribution to the genomic repertoire of $T$. brucei, relative to other trypanosomatids, and was a seminal development in its genomic evolution.

\section{Methods}

\section{(a) Examination of the duplicated regions}

The extent of colinearity between Chromosomes 4 and 8 in T. brucei was assessed using the Artemis Comparison Tool v5 (ACT [74]). A tBLASTx algorithm [75] was used to create a sequence comparison from EMBL files of the two chromosomes. The duplicated regions of Chromosomes 4 and 8 were inspected visually from the GeneDB chromosome maps page [76], to determine the gene order on each duplicon and affinity shown by each paralogous gene pair. Sequence identity was calculated as the proportion of amino acids conserved when paralogous genes were aligned in BioEdit [77]. Inspection of homologs in Leishmania major showed that the duplicated region in $T$. brucei corresponded to the complete Chromosome 31 in L. major. The gene order in L. major (and another related trypanosome, T. cruzi) was used to infer the gene order on the chromosome ancestral to the duplicons on Chromosomes 4 and 8 in T. brucei. If present on both duplicons and either outgroup, a locus was shared, i.e., originally single-copy and now retained as two paralogs. If present on one duplicon and either outgroup, the locus was con- sidered to have been present on the ancestral chromosome and lost after duplication from the either duplicon. If present on one duplicon but absent from either outgroup, this was interpreted as gain of a locus after duplication. Situations were both duplicons retained a locus that was absent in both $\mathrm{L}$. major and $T$. cruzi were not observed. The duplicated regions in T. brucei were also compared to homologous regions in its closest relative, $T$. congolense. If it could be shown that $T$. congolense possessed duplicate copies for the loci in these regions, this would show that the duplication occurred prior to the separation of these two species. Gene order along the $T$. brucei duplicons was compared with preliminary assemblies for chromosomes 4 and 8 in T. congolense (available from GeneDB).

\section{(b) Analysis of divergence of paralogous sequences}

Patterns in sequence divergence post-duplication were analysed by comparing the identity shown by coding regions of paralogous gene pairs with that shown by noncoding regions. Untranslated regions of genes were identified from genomic sequence in Artemis v8 [78] after identifying the sequence motifs for spliced leader and poly-A tail additions, established previously [79]. These motifs signal the creation of individual transcripts from nascent polycistronic transcripts; they are arranged in a fairly consistent manner, with a polypyrimidine tract providing the signal for poly-A tail addition (i.e., the end of the 3' UTR), and the next downstream AG dinucleotide signalling for the addition of the spliced leader sequence (i.e., the start of the 5' UTR) [79]. Determination of these points for each paralogous gene pair allowed sequence identity to be calculated for coding, 5'UTR and 3'UTR regions respectively. If paralogous non-coding regions could be aligned, nucleotide identity was calculated from the alignment; if not, a nominal value was calculated from unaligned sequences. In both cases, sequences were trimmed to equal length since length differences would reduce sequence identity overall.

\section{(c) Relative rates tests}

Significant departures in evolutionary rate post-duplication were identified using the relative rates test $[80,81]$. Paralogous gene pairs were combined in a sequence alignment with a homolog from $T$. cruzi (or where this was absent, T. vivax), retrieved from GeneDB, and designated as an outgroup comparison. The rates of non-synonymous substitutions per site $\left(D_{n}\right)$ between each paralog and an outgroup were compared using RRTree [82]; synonymous substitutions were not compared since these were frequently saturated over the relatively large evolutionary distances concerned. Due to the various weaknesses of the canonical, i.e., Wu-Li, relative rates test, a Bayesian approach was adopted as previously described [83]). The genetic distance between each paralog and the 
most recent common ancestor of all three sequences was estimated using Cadence [83]; this was calculated from 5000 Bayesian phylogenies to estimate 95\% confidence intervals. Significant differences in evolutionary rate were inferred where these confidence intervals did not overlap.

\section{(d) Tests for non-neutral evolution}

Paralogous CDS sequences were analysed for evidence of adaptive evolution using two methods. First, alignments were generated for each pair of paralogous sequences and for each singleton locus along the duplicated region, in combination with their $T$. congolense homologs. The latter were included to allow the singleton loci to be analysed and directly compared with conserved paralogs. The ratio of non-synonymous substitutions per site $\left(D_{n}\right)$ to synonymous substitutions per site $\left(D_{s}\right)$, referred to as $\omega$, was calculated for each codon of each alignment using the adaptive evolution server [84], within the HYPHY platform [85]. Three methods were used to detect both positive and negative selection at individual codons: i) SingleLikelihood-Ancestor-Counting (SLAC), ii) Fixed-EffectLikelihood (FEL) and iii) Random-Effects-Likelihood (REL), which have been described in detail elsewhere [86]. The inclusion of a T. congolense homolog as an outgroup sequence could introduce or obscure further evidence for selection, hence, conserved paralogs were tested again without an outgroup, using the REL method.

Second, paralogous sequences were aligned with homologs from related trypanosomatids ( $T$. congolense, $T$. vivax, T. cruzi, as appropriate and where available) and used to create a phylogenetic tree and reconstruct ancestral sequences. This was done using CRANN [87], which applies the method of [88]. The frequencies of invariant (i.e., change once but not again) and variable (change frequently) mutations at both non-synonymous and synonymous sites were calculated along the branches leading to each paralog from their inferred ancestor. The ratios of invariant to variable mutations at non-synonymous and synonymous sites were calculated; significant differences between these values were identified using a G-test. Significant differences due to an excess of invariant mutations at non-synonymous sites ('replacement-invariable', or RI) indicate adaptive change $[89,90]$, in essence because the ratio at synonymous sites represents the expectation under neutral conditions, and the ratio at non-synonymous sites should be not significantly different in the absence of positive selection.

\section{(e) Taxonomic distribution of the duplicons}

Observation and analysis of the duplication event was made from the genome sequence of $T$. brucei brucei strain 927. A polymerase chain reaction (PCR) assay was applied to determine whether the duplication was also present in other strains of T. brucei, namely T. b. brucei (TSW 187), T. b. gambiense (Dal 972), T. b. rhodiense (LVH 108) and T. evansi (RoTat 1.2). Three locations along the duplicated region were selected where there was a shared paralog followed downstream by distinct single-copy genes (i.e., lost from one chromosome) on each duplicon. For each location, a common forward primer and dissimilar reverse primers were used to amplify the two distinct intergenic regions from each T. brucei subspecies. Successful amplification of products with expected size confirmed that both duplicons were present. Genomic DNA was denatured at $92^{\circ} \mathrm{C}$ for 2 minutes and then 35 amplification cycles were performed under the following conditions: denaturation at $92^{\circ} \mathrm{C}$ (30 seconds), annealing at $60^{\circ} \mathrm{C}$ (10 seconds) and extension at $72{ }^{\circ} \mathrm{C}$ (90 seconds). The locus, forward primer, chromosome 4 -specific reverse primer (with expected product size), and chromosome 8-specific reverse primer (with expected product size) are given for each location in turn. Location 1: 14 (Tb927.4.5180 and Tb927.8.7220); 1F, TGCAACTCAGTCAGGACCCGT; 1R4 (1310 bp), TCCCAGCAACACCTTCAGTTT; 1R8 (1929 bp), TAACATTTCCACCGCTACCTG. Location 2: 25 (Tb927.4.4990 and Tb927.8.7430); 2F, GAGCGCATCAAGGATATCCCT; 2R4 (1180 bp), GCCTCCATCAATGTTAAACCA; 2R8 (2039 bp), CTTCAAGACGAACGCAG ACTC. Location 3: 55 (Tb927.4.4330 and Tb927.8.8040); 3F, GGTCCTGAAACGGTGGTGTTT; 3R4 (1030 bp), CGTGCTGTATGGGTGATTCTT; 3R8 (1560 bp), ACAAGAAGAATGTGCCACCAC.

\section{Abbreviations}

ACT Artemis Comparison Tool

CDS Coding sequence

FEL Fixed effects likelihood

NCS Non-coding sequence

PCR Polymerase chain reaction

REL Random effects likelihood

RI 'Replacement-invariable' mutation

SLAC Single-likelihood ancestor counting

UTR Untranscribed region

VSG Variant surface glycoprotein

WGD Whole genome duplication 


\section{Additional material}

\section{Additional data file 1}

Figure S1. Comparative gene order and sequence identity between duplicons. Chromosome 4 is shown above, and running antiparallel to, chromosome 8. Scale is shown in Mbp and corresponds to positions shown on chromosome maps in GeneDB. Genes are marked on chromosomes in three colours: grey (shared paralogs on both duplicons, numbered 1 to 74), red (unilaterally lost from one duplicon) and green (unilaterally gained after duplication by one duplicon). All genes may be clicked to link to positions and gene models in GeneDB. Paralogs are linked by shaded bars that reflect amino acid sequence identity. Loci with positive identifications in GeneDB are labelled by gene name. Coloured arrows relate to significant results in sequence analyses and may be clicked to link to relevant data tables: blue (significant asymmetry in canonical relative rates test), dark blue (significant asymmetry in Bayesian relative rates test), green (significant difference in the invariable-variable mutation ratios at non-synonymous vs. synonymous sites) and red $(\omega>1)$.

Click here for file

[http://www.biomedcentral.com/content/supplementary/14712164-8-432-S1.png]

\section{Additional data file 2}

Table S1. Paralogs retained on both duplicons: inter-chromosomal and interspecific sequence identity in CDS and NCS regions and UTR length. Click here for file

[http://www.biomedcentral.com/content/supplementary/1471-

2164-8-432-S2.doc]

\section{Additional data file 3}

Table S2. Results of canonical relative rates tests, using the non-synonymous substitution rate per site $\left(\mathrm{D}_{\mathrm{n}}\right)$ since duplication, on shared, paralogous CDSs.

Click here for file

[http://www.biomedcentral.com/content/supplementary/14712164-8-432-S3.doc]

\section{Additional data file 4}

Table S3. Results of Bayesian relative rates tests on shared paralogs, comparing total genetic distance to MRCA.

Click here for file

[http://www.biomedcentral.com/content/supplementary/1471-

2164-8-432-S4.doc]

\section{Additional data file 5}

Table S4. Evidence for positive and negative selection per codon for singlecopy loci (i.e., duplicate lost) and retained paralogs within the duplicated region.

Click here for file

[http://www.biomedcentral.com/content/supplementary/14712164-8-432-S5.doc]

\section{Additional data file 6}

Table S5. Comparisons of ratios of invariable to variable mutations at synonymous (i.e., silent, $S$ ) and non-synonymous (i.e., replacement, $R$ ) sites, between shared paralogs.

Click here for file

[http://www.biomedcentral.com/content/supplementary/14712164-8-432-S6.doc]

\section{Additional data file 7}

Figure S2. PCR assay to determine the taxonomic distribution of the duplication event. To confirm the presence of both duplicons in four subspecies of $\mathrm{T}$. brucei (brucei (Tbb), rhodiense (Tbr), gambiense (Tbg) and evansi (Tev)], three locations along the duplicated region (1-3, shown with corresponding GeneDB identifiers) were selected. In each case, a shared paralog was followed downstream by dissimilar single-copy genes on the different duplicons. Amplification of the two dissimilar intergenic regions for each location (shown at right with primer names and expected PCR products sizes) yielded the expected products from all four subspecies.

Click here for file

[http://www.biomedcentral.com/content/supplementary/14712164-8-432-S7.png]

\section{Acknowledgements}

This work was funded by the Wellcome Trust. Internet support for the supplementary figure was provided by Adrian Tivey. Genome sequence data were generated by the Pathogen Sequencing Unit of the Wellcome Trust Sanger Institute and funded by the Wellcome Trust. Trypanosome genomic DNA was kindly provided by Wendy Gibson (University of Bristol) and Philippe Büscher (Instituut voor Tropische Geneeskunde, Belgium). APJ is a Wellcome Trust Sanger Institute Post-doctoral Fellow.

\section{References}

I. Berriman M, Ghedin E, Hertz-Fowler C, Blandin G, Renauld H, Bartholomeu DC, Lennard NJ, Caler E, Hamlin NE, Haas B, Böhme U, Hannick L, Aslett MA, Shallom J, Marcello L, Hou L, Wickstead B, Alsmark UC, Arrowsmith C, Atkin RJ, Barron AJ, Bringaud F, Brooks K, Carrington M, Cherevach I, Chillingworth TJ, Churcher C, Clark LN, Corton $\mathrm{CH}$, Cronin A, Davies RM, Doggett J, Diikeng A, Feldblyum T, Field MC, Fraser A, Goodhead I, Hance Z, Harper D, Harris BR, Hauser $H$, Hostetler J, Ivens A, Jagels K, Johnson D, Johnson J, Jones K, Kerhornou AX, Koo H, Larke N, Landfear S, Larkin C, Leech V, Line A, Lord A, Macleod A, Mooney PJ, Moule S, Martin DM, Morgan GW, Mungall K, Norbertczak H, Ormond D, Pai G, Peacock CS, Peterson J, Quail MA, Rabbinowitsch E, Rajandream MA, Reitter C, Salzberg SL, Sanders M, Schobel S, Sharp S, Simmonds M, Simpson AJ, Tallon L, Turner CM, Tait A, Tivey AR, Van Aken S, Walker D, Wanless D, Wang S, White B, White O, Whitehead S, Woodward I, Wortman J, Adams MD, Embley TM, Gull K, Ullu E, Barry JD, Fairlamb AH, Opperdoes F, Barrell BG, Donelson JE, Hall N, Fraser CM, Melville SE, El-Sayed NM: The genome of the African trypanosome Trypanosoma brucei. Science 2005, 309:416-422.

2. El-Sayed NM, Myler PJ, Bartholomeu DC, Nilsson D, Aggarwal G, Tran AN, Ghedin E, Worthey EA, Delcher AL, Blandin G, Westenberger S], Caler E, Cerqueira GC, Branche C, Haas B, Anupama A, Arner E, Aslund L, Attipoe P, Bontempi E, Bringaud F, Burton $\mathrm{P}$, Cadag E, Campbell DA, Carrington M, Crabtree J, Darban H, da Silveira JF, de Jong P, Edwards K, Englund PT, Fazelina G, Feldblyum T, Ferella M, Frasch AC, Gull K, Horn D, Hou L, Huang Y, Kindlund E, Klingbeil M, Kluge S, Koo H, Lacerda D, Levin MJ, Lorenzi H, Louie T, Machado CR, McCulloch R, McKenna A, Mizuno Y, Mottram JC, Nelson S, Ochaya S, Osoegawa K, Pai G, Parsons M, Pentony M, Pettersson U, Pop M, Ramirez JL, Rinta J, Robertson L, Salzberg SL, Sanchez DO, Seyler A, Sharma R, Shetty J, Simpson AJ, Sisk E, Tammi MT, Tarleton R, Teixeira S, Van Aken S, Vogt C, Ward PN, Wickstead B, Wortman J, White O, Fraser CM, Stuart KD, Andersson B: The genome sequence of Trypanosoma cruzi, etiologic agent of Chagas disease. Science 2005, 309:409-4I5.

3. Ivens AC, Peacock CS, Worthey EA, Murphy L, Aggarwal G, Berriman M, Sisk E, Rajandream MA, Adlem E, Aert R, Anupama A, Apostolou Z, Attipoe P, Bason N, Bauser C, Beck A, Beverley SM, Bianchettin G, Borzym K, Bothe G, Bruschi CV, Collins M, Cadag E, Ciarloni L, Clayton C, Coulson RM, Cronin A, Cruz AK, Davies RM, De Gaudenzi J, Dobson DE, Duesterhoeft A, Fazelina G, Fosker N, Frasch AC, Fraser A, Fuchs M, Gabel C, Goble A, Goffeau A, Harris D, Hertz-Fowler C, 
Hilbert H, Horn D, Huang Y, Klages S, Knights A, Kube M, Larke N, Litvin L, Lord A, Louie T, Marra M, Masuy D, Matthews K, Michaeli S, Mottram JC, Müller-Auer S, Munden H, Nelson S, Norbertczak H, Oliver K, O'neil S, Pentony M, Pohl TM, Price C, Purnelle B, Quail MA, Rabbinowitsch E, Reinhardt R, Rieger M, Rinta J, Robben J, Robertson L, Ruiz JC, Rutter S, Saunders D, Schäfer M, Schein J, Schwartz DC, Seeger K, Seyler A, Sharp S, Shin H, Sivam D, Squares R, Squares S, Tosato V, Vogt C, Volckaert G, Wambutt R, Warren T, Wedler $H$, Woodward J, Zhou S, Zimmermann W, Smith DF, Blackwell JM, Stuart KD, Barrell B, Myler PJ: The genome of the kinetoplastid parasite, Leishmania major. Science 2005, 309:436-442.

4. Bastien P, Blaineau C, Pages M: Molecular karyotype analysis in Leishmania. Subcell Biochem 1992, I8:131-87.

5. Melville SE, Gerrard CS, Blackwell JM: Multiple causes of size variation in the diploid megabase chromosomes of African tyrpanosomes. Chromosome Res 1999, 7:191-203.

6. Hope M, MacLeod A, Leech V, Melville S, Sasse J, Tait A, Turner CM: Analysis of ploidy (in megabase chromosomes) in Trypanosoma brucei after genetic exchange. Mol Biochem Parasitol 1999, 104:1-9.

7. Campbell DA, Westenberger SJ, Sturm NR: The determinants of Chagas disease: connecting parasite and host genetics. Curr Mol Med 2004, 4:549-62.

8. Martinez-Calvillo S, Stuart K, Myler PJ: Ploidy changes associated with disruption of two adjacent genes on Leishmania major chromosome I. Int J Parasitol 2005, 35:4I9-29.

9. Melville SE, Leech V, Gerrard CS, Tait A, Blackwell JM: The molecular karyotype of the megabase chromosomes of Trypanosoma brucei and the assignment of chromosome markers. Mol Biochem Parasitol 1998, 94:155-73.

10. El-Sayed NM, Myler PJ, Blandin G, Berriman M, Crabtree J, Aggarwal G, Caler E, Renauld H, Worthey EA, Hertz-Fowler C, Ghedin E, Peacock C, Bartholomeu DC, Haas BJ, Tran AN, Wortman JR, Alsmark UC, Angiuoli S, Anupama A, Badger J, Bringaud F, Cadag E, Carlton JM, Cerqueira GC, Creasy T, Delcher AL, Djikeng A, Embley TM, Hauser C, Ivens AC, Kummerfeld SK, Pereira-Leal JB, Nilsson D, Peterson J, Salzberg SL, Shallom J, Silva JC, Sundaram J, Westenberger S, White O, Melville SE, Donelson JE, Andersson B, Stuart KD, Hall $\mathrm{N}$ : Comparative genomics of trypanosomatid parasitic protozoa. Science 2005, 309:404-409.

II. Van der Peer Y, Meyer A: Large-scale gene and ancient genome duplications. In The Evolution of the Genome Edited by: Gregory TR. Burlington, MA: Elsevier; 2005:330-37I.

12. Taylor JS, Raes J: Small-scale gene duplications. In The Evolution of the Genome Edited by: Gregory TR. Burlington, MA: Elsevier; 2005:290-329.

13. Jin H, Selfe J, Whitehouse C, Morris JR, Solomon E, Roberts RG: Structural evolution of the BRCAl genomic region in primates. Genomics 2004, 84: I07|-82.

14. Simillion C, Vandepoele K, Van Montagu MC, Zabeau M, Van de Peer Y: The hidden duplication past of Arabidopsis thaliana. Proc Natl Acad Sci USA 2002, 99: I 3627-32.

15. Tate JA, Soltis DE, Soltis PS: Polyploidy in plants. In The Evolution of the Genome Edited by: Gregory TR. Burlington, MA: Elsevier; 2005:372-427.

16. De Bodt S, Maere S, Van de Peer Y: Genome duplication and the origin of angiosperms. Trends Ecol Evol 2005, 20:59I-597.

17. Yu J, Wang J, Lin W, Li S, Li H, Zhou J, Ni P, Dong W, Hu S, Zeng C, Zhang J, Zhang Y, Li R, Xu Z, Li S, Li X, Zheng H, Cong L, Lin L, Yin J, Geng J, Li G, Shi J, Liu J, Lv H, Li J, Wang J, Deng Y, Ran L, Shi X, Wang X, Wu Q, Li C, Ren X, Wang J, Wang X, Li D, Liu D, Zhang X, Ji Z, Zhao W, Sun Y, Zhang Z, Bao J, Han Y, Dong L, Ji J, Chen P, Wu S, Liu J, Xiao Y, Bu D, Tan J, Yang L, Ye C, Zhang J, Xu J, Zhou Y, Yu Y, Zhang B, Zhuang S, Wei H, Liu B, Lei M, Yu H, Li Y, Xu H, Wei S, He X, Fang L, Zhang Z, Zhang Y, Huang X, Su Z, Tong W, Li J, Tong Z, Li S, Ye J, Wang L, Fang L, Lei T, Chen C, Chen H, Xu Z, Li H, Huang H, Zhang F, Xu H, Li N, Zhao C, Li S, Dong L, Huang Y, Li L, Xi Y, Qi Q, Li W, Zhang B, Hu W, Zhang Y, Tian X, Jiao Y, Liang X, Jin J, Gao L, Zheng W, Hao B, Liu S, Wang W, Yuan L, Cao M, McDermott J, Samudrala R, Wang J, Wong GK, Yang H: The Genomes of Oryza sativa : a history of duplications. PLoS Biol 2005, 3:e38.

18. Wolfe KH, Shields DC: Molecular evidence for an ancient duplication of the entire yeast genome. Nature 1997, 387:708-713.

19. Kellis M, Birren BW, Lander ES: Proof and evolutionaryanalysis of ancient genome duplication in the yeast Saccharomyces cerevisiae. Nature 2004, 428:617-624.
20. Ochman H, Daubin V, Lerat E: A bunch of fun-guys: the wholegenome view of yeast evolution. Trends Genet 2005, 2 I: I-3.

21. Liti G, Louis EJ: Yeast evolution and comparative genomics. Annu Rev Microbiol 2005, 59: I35-I53.

22. Wolfe KH: Yesterday's polyploids and the mystery of diploidization. Nat Rev Genet 200I, 2:333-34I.

23. Durand D: Vertebrate evolution: doubling and shuffling with a full deck. Trends Genet 2003, 19:2-5.

24. Blomme T, Vandepoele K, De Bodt S, Simillion C, Maere S, Van de Peer $Y$ : The gain and loss of genes during $\mathbf{6 0 0}$ million years of vertebrate evolution. Genome Biol 2006, 7:R43.

25. Ohno S: Evolution by gene duplication. New York: Springer-Verlag; 1970.

26. Blanc G, Wolfe KH: Functional divergence of duplicated genes formed by polyploidy during Arabidopsis evolution. Plant Cell 2004, 16:1679-91.

27. Paterson AH, Chapman BA, Kissinger JC, Bowers JE, Feltus FA, Estill $\mathrm{JC}$ : Many gene and domain families have convergent fates following independent whole-genome duplication events in Arabidopsis, Oryza, Saccharomyces and Tetraodon. Trends Genet 2006, 22:597-602.

28. Brunet FG, Crollius HR, Paris M, Aury JM, Gibert P, Jaillon O, Laudet $\checkmark$, Robinson-Rechavi M: Gene loss and evolutionary rates following whole-genome duplication in teleost fishes. Mol Biol Evol 2006, 23:1808-16.

29. Tian CG, Xiong YQ, Liu TY, Sun SH, Chen LB, Chen MS: Evidence for an ancient whole-genome duplication event in rice and other cereals. Yi Chuan Xue Bao 2005, 32:519-27.

30. Conant GC, Wagner A: Asymmetric sequence divergence of duplicate genes. Genome Res 2003, 13:2052-2058.

3I. Kim SH, Yi SV: Correlated asymmetry of sequence and functional divergence between duplicate proteins of Saccharomyces cerevisiae. Mol Biol Evol 2006, 23:1068-1075.

32. Byrne KP, Wolfe KH: Consistent patterns of rate asymmetry and gene loss indicate widespread neofunctionalization of yeast genes after whole-genome duplication. Genetics 2007, I75: $134 \mid-50$.

33. Wang H, Yu L, Lai F, Liu L, Wang J: Molecular evidence for asymmetric evolution of sister duplicated blocks after cereal polyploidy. Plant Mol Biol 2005, 59:63-74.

34. Jiang H, Liu D, Gu Z, Wang W: Rapid evolution in a pair of recent duplicate segments of rice. J Exp Zoolog B Mol Dev Evol 2007, 308:50-7.

35. Dulai KS, von Dornum M, Mollon JD, Hunt DM: The evolution of trichromatic color vision by opsin gene duplication in New World and Old World primates. Genome Res 1999, 9:629-38.

36. Zhang J, Zhang YP, Rosenberg HF: Adaptive evolution of a duplicated pancreatic ribonuclease gene in a leaf-eating monkey. Nat Genet 2002, 30:4II-5.

37. Hughes AL: The evolution of functionally novel proteins after gene duplication. Proc Biol Sci 1994, 256: I 19-24.

38. Force A, Lynch M, Pickett FB, Amores A, Yan YL, Postlethwait J: Preservation of duplicate genes by complementary, degenerative mutations. Genetics 1999, 151:1531-1545.

39. Lynch M, Conery JS: The evolutionary fate and consequences of duplicate genes. Science 2000, 290: II5I-II55.

40. Lynch M, Conery JS: The evolutionary demography of duplicate genes. J Struct Funct Genomics 2003, 3:35-44.

4I. Zhang P, Chopra S, Peterson T: Segmental gene duplication generated differentially expressed myb -homologous genes in maize. Plant Cell 2000, I 2:23 II-2322.

42. Tümpel S, Cambronero F, Wiedemann LM, Krumlauf R: Evolution of cis elements in the differential expression of two Hoxa2 coparalogous genes in pufferfish (Takifugu rubripes). Proc Nat Acad Sci USA 2006, 103:5419-24.

43. Roth C, Rastogi S, Arvestad L, Dittmar K, Light S, Ekman D, Liberles DA: Evolution after gene duplication: models, mechanisms, sequences, systems, and organisms. J Exp Zoolog B Mol Dev Evol 2007, 308:58-73.

44. Le Blancq SM, Swinkels BW, Gibson WC, Borst P: Evidence for gene conversion between the phosphoglycerate kinase genes of Trypanosoma brucei. J Mol Biol 1988, 200:439-447.

45. Bringaud F, Baltz T: African trypanosome glucose transporter genes: organization and evolution of a multigene family. Mol Biol Evol 1994, I I:220-230. 
46. Jackson AP: Tandem gene arrays in Trypanosoma brucei : comparative phylogenomic analysis of duplicate sequence variation. BMC Evol Biol 2007, 7:54.

47. Rochette A, McNicoll F, Girard J, Breton M, Leblanc E, Bergeron MG Papadopoulou B: Characterization and developmental gene regulation of a large gene family encoding amastin surface proteins in Leishmania spp. Mol Biochem Parasitol 2006 , | 40:205-220.

48. Taylor JE, Rudenko G: Switching trypanosome coats: what's in the wardrobe? Trends Genet 2006, 22:6|4-620.

49. Interactive figure showing alignment of duplicatedregions in T. brucei [http://www.genedb.org/genedb/tryp/48dup image.jsp]

50. Gibson W, Garside L, Bailey M: Trisomy and chromosome size changes in hybrid trypanosomes from a genetic cross between Trypanosoma brucei rhodesiense and T. b. brucei. Mo Biochem Parasitol 1992, 5 I: 189-99.

5I. Hughes AL: Phylogenies of developmentally important proteins do not support the hypothesis of two rounds of genome duplication early in vertebrate history. J Mol Evol 1999, 48:565-76.

52. Hughes AL, Friedman R: 2R or not 2R: testing hypotheses of genome duplication in early vertebrates. J Struct Funct Genomics 2003, 3:85-93.

53. Hughes AL, Friedman R: Pattern of divergence of amino acid sequences encoded by paralogous genes in human and pufferfish. Mol Phylogenet Evol 2004, 32:337-43.

54. Furlong RF, Holland PW: Were vertebrates octoploid? Philos Trans R Soc Lond B Biol Sci 2002, 357:531-44.

55. McLysaght A, Hokamp K, Wolfe $\mathrm{KH}$ : Extensive genomic duplication during early chordate evolution. Nat Genet 2002, 3 I :200-4

56. Koszul R, Caburet S, Dujon B, Fischer G: Eucaryotic genome evolution through the spontaneous duplication of large chromosomal segments. EMBO J 2004, 23:234-43.

57. Llorente B, Durrens $P$, Malpertuy A, Aigle M, Artiguenave F, Blandin G, Bolotin-Fukuhara M, Bon E, Brottier P, Casaregola S, Dujon B, de Montigny J, Lépingle A, Neuvéglise C, Ozier-Kalogeropoulos O, Potier S, Saurin W, Tekaia F, Toffano-Nioche C, Wésolowski-Louve M, Wincker P, Weissenbach J, Souciet J, Gaillardin C: Genomic exploration of the hemiascomycetous yeasts: I 8. Comparative analysis of chromosome maps and synteny with Saccharomyces cerevisiae. FEBS Lett 2000, 487:101-I2.

58. Cha RS, Kleckner N: ATR homolog Mecl promotes fork progression, thus averting breaks in replication slow zones. Science 2002, 297:602-6.

59. Pelletier R, Krasilnikova MM, Samadashwily GM, Lahue R, Mirkin SM Replication and expansion of trinucleotide repeats in yeast. Mol Cell Biol 2003, 23: I349-57.

60. Thomas BC, Pedersen B, Freeling M: Following tetraploidy in an Arabidopsis ancestor, genes were removed preferentially from one homeolog leaving clusters enriched in dose-sensitive genes. Genome Res 2006, I 6:934-46.

61. Cliften PF, Fulton RS, Wilson RK, Johnston M: After the duplication: gene loss and adaptation in Saccharomyces genomes. Genetics 2006, I 72:863-872.

62. Lin YS, Hwang JK, Li WH: Protein complexity, gene duplicability and gene dispensability in the yeast genome. Gene 2007, 387: $109-17$

63. Papp B, Csaba P, Hurst LL: Dosage sensitivity and the evolution of gene families in yeast. Nature 2003, 424: 194-7.

64. Birchler JA, Riddle NC, Auger DL, Veitia RA: Dosage balance in gene regulation: biological implications. Trends Genet 2005 2I:219-26.

65. Yang J, Lusk R, Li WH: Organismal complexity, protein complexity, and gene duplicability. Proc Natl Acad Sci USA 2003 , 100: |566|-5.

66. Prachumwat A, Li WH: Protein function, connectivity, and duplicability in yeast. Mol Biol Evol 2006, 23:30-9.

67. Burhans DT, Ramachandran L, Wang J, Liang P, Patterton HG Breitenbach $M$, Burhans WC: Non-random clustering of stressrelated genesduring evolution of the $S$. cerevisiae genome. BMC Evol Biol 2006, 6:58.

68. Davis JC, Petrov DA: Preferential duplication of conserved proteins in eukaryotic genomes. PLoS Biol 2004, 2:E55.

69. Chen L, DeVries AL, Cheng $\mathrm{CH}$ : Evolution of antifreeze glycoprotein gene from a trypsinogen gene in Antarctic notothenioid fish. Proc Natl Acad Sci USA 1997, 94:38 I I-6.
70. Bhushan S, Stahl A, Nilsson S, Lefebvre B, Seki M, Roth C, McWilliam D, Wright SJ, Liberales DA, Shinozaki K, Bruce BD, Boutry M, Glaser $\mathrm{E}$ : Catalysis, subcellular localization, expression and evolution of the targeting peptides degrading protease, AtPreP2. Plant Cell Physiol 2005, 46:985-96.

7I. Hua LV, Hidaka K, Pesesse X, Barnes LD, Shears SB: Paralogous murine Nudt I 0 and Nudt I I genes have differential expression patterns but encode identical proteins that are physiologically competent diphosphoinositol polyphosphate phosphohydrolases. Biochem J 2003, 373:8I-9.

72. Averof M, Dawes R, Ferrier D: Diversification of arthropod Hox genes as a paradigm for the evolution of gene functions. Sem Cell Devel Biol 1996, 4:539-55I.

73. McClintock JM, Carlson R, Mann DM, Prince VE: Consequences of Hox gene duplication in the vertebrates: an investigation of the zebrafish Hox paralogue group I genes. Development 200 I, | 28:247|-84

74. Carver T], Rutherford KM, Berriman M, Rajandream MA, Barrell BG, Parkhill J: ACT: the Artemis Comparison Tool. Bioinformatics 2005, 2 I:3422-3423.

75. Altschul S, Boguski MS, Gish W, Wootton JC: Issues in searching molecular sequence database. Nat Genet 1994, 6: I I9- I 29.

76. Wellcome Trust Sanger Institute, PathogenSequencing Unit 'GeneDB' Interface [http://www.genedb.org/tryp/]

77. Martin DP, Williamson C, Posada D: RDP2: recombination detection and analysis from sequence alignments. Bioinformatics 2005, $21: 260-262$

78. Rutherford K, Parkhill J, Crook J, Horsnell T, Rice P, Rajandream MA, Barrell B: Artemis: sequence visualization and annotation. Bioinformatics 2000, I 6:944-945.

79. Benz C, Nilsson D, Andersson B, Clayton C, Guilbride DL: Messenger RNA processing sites in Trypanosoma brucei. Mol Biochem Parasitol 2005, I 43: I25-134.

80. $\mathrm{Wu} \mathrm{Cl}$, $\mathrm{ii} \mathrm{WH}$ : Evidence for higher rates of nucleotide substitution in rodents than in man. Proc Natl Acad Sci USA 1985, 82: $174 \mid-1745$.

81. Tajima F: Simple methods for testing the molecular evolutionary clock hypothesis. Genetics 1993, I 35:599-607.

82. Robinson-Rechavi $M$, Huchon $D$ : RRTree: relative-rate tests between groups of sequences on a phylogenetic tree. Bioinformatics 2000, 16:296-7.

83. Wilcox TP, Garcia de Leon FJ, Hendrickson DA, Hillis DM: Conver gence among cave catfishes: long-branch attraction and a Bayesian relative rates test. Mol Phylogenet Evol 2004, 3 I:II0I-I| I3.

84. Kosakovsky Pond SL, Frost SDW: Datamonkey: rapid detection of selective pressure on individual sites of codon alignments. Bioinformatics 2005, 21:2531-2533.

85. Kosakovsky Pond SL, Frost SDW, Muse SV: HyPhy: hypothesis testing using phylogenies. Bioinformatics 2005, 21 :676-679.

86. Kosakovsky Pond SL, Frost SDW: Not so different after all: a comparison of methods for detecting amino acid sites under selection. Mol Biol Evol 2005, 22: I208-1222.

87. Creevey CJ, Mclnerney JO: CRANN: detecting adaptive evolution in protein-coding DNA sequences. Bioinformatics 2003 19:1726.

88. McDonald $\mathrm{JH}$, Kreitman M: Adaptive protein evolution at the Adh locus in Drosophila. Nature 1991, 35 I:652-654.

89. Creevey CJ, Mclnerney JO: An algorithm for detecting directional and non-directional positive selection, neutrality and negative selection in protein coding DNA sequences. Gene 2002, 300:43-51.

90. Gribaldo S, Casane D, Lopez P, Philippe H: Functional divergence prediction from evolutionary analysis: a case study of vertebrate hemoglobin. Mol Biol Evol 2003, 20: I754-I759. 